\title{
What do the Engel laws and positive laws have in common
}

\author{
Olga Macedonska \\ Institute of Mathematics, Silesian University of Technology, \\ Gliwice 44-100, Poland \\ e-mail: o.macedonska@polsl.pl
}

\begin{abstract}
Аннотация
Статья связана с вопросом Р. Бернса: Что общего имеют Энгелевые и полугрупповые тождества, заставляя конечно порожденные локально ступенчатые группы содержать нильпотентную подгруппу конечного индекса? Мы показываем, что Энгелевые и полугрупповые тождества имеют одинаковую так называемую Энгелевую конструкцию, а каждая конечно порожденная локально ступенчатая группа удовлетворяющая тождеству с такой конструкцией должна содержать нильпотентную подгруппу конечного индекса.
\end{abstract}

\begin{abstract}
The work is inspired by a question of R.Burns: What do the Engel laws and positive laws have in common that forces finitely generated locally graded groups satisfying them to be nilpotent-by-finite? The answer is that these laws have the same so called the Engel construction.
\end{abstract}

\section{Introduction}

Let $F=\langle x, y\rangle$ be a free group of rank $2, u$ be a word, and $S$ be a subset in $F$.

Definition 1. We say that a law $w \equiv 1$ has construction $u \widetilde{\in} S$ if it is equivalent to a law $u \equiv s$ for some $s \in S$. 
The laws with the same construction have similar properties. For example, the laws with construction $[x, y] \widetilde{\in} F^{\prime \prime}$ force the groups satisfying them to have perfect commutator subgroups.

We denote $x^{y^{i}}=y^{-i} x y^{i},[x, y]=x^{-1} y^{-1} x y, \quad\left[x,{ }_{i} y\right]$ is an Engel commutator $[\ldots[[x, y], y], \ldots, y]$ where $y$ is repeated $i$ times, and $\left[x,{ }_{0} y\right]=x$. By $E_{n}$ we denote the following subgroup generated by the Engel commutators:

$$
E_{n}=\left\langle\left[x,{ }_{i} y\right], 0 \leq i \leq n\right\rangle .
$$

We show that every binary commutator law is equivalent to a law with the following so called Engel construction

$$
[x, y]^{k_{1}}\left[x,{ }_{2} y\right]^{k_{2}} \ldots\left[x,{ }_{n} y\right]^{k_{n}} \widetilde{\in} E_{n}^{\prime} .
$$

Let $w \equiv 1$ be a binary law and $\mathfrak{V}$ be a variety, it defines. We prove that

- Each finitely generated group in $\mathfrak{V}$ has finitely generated commutator subgroup if and only if the law $w \equiv 1$ implies a law with the following Engel construction

$$
\left[x,{ }_{n} y\right] \widetilde{\in} E_{n-1} .
$$

- Positive laws and the Engel laws have the Engel construction (1). The law $x^{k} \equiv 1$ implies a law with the Engel construction (1).

- Each finitely generated locally graded group satisfying a law with the Engel construction (1) is nilpotent-by-finite.

\section{The Engel construction of laws}

We show that every binary commutator law is equivalent to a law $w \equiv$ 1 , where for some $n$, the word $w$ is a product of the Engel commutators $\left[x,{ }_{i} y\right], 1 \leq i \leq n$.

Theorem 1. Every binary commutator law $w \equiv 1$ has the following Engel construction

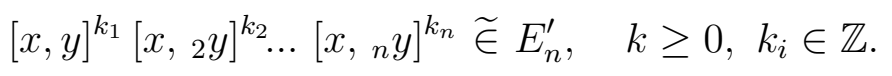


Proof. Let $w \equiv 1$ be a commutator law. Note that $F^{\prime}$ belongs to the normal closure of $x$ in $F$ which is freely generated by all conjugates $x^{y^{i}}, i \in \mathbb{Z}$. So $w$ is a product of some $x^{y^{i}}$ with say, $-m \leq i \leq-m+n$. Conjugation by $y^{m}$ gives us the equivalent law with $w \in\left\langle x^{y^{i}}, 0 \leq i \leq n\right\rangle$. In this subgroup we can replace the free generators $x^{y^{i}}$ by $x^{-1} x^{y^{i}}=\left[x, y^{i}\right]$, then

$$
w \in\left\langle x^{y^{i}}, 0 \leq i \leq n\right\rangle=\left\langle x,\left[x, y^{i}\right], 1 \leq i \leq n\right\rangle .
$$

We show by induction that $\left\langle x,\left[x, y^{i}\right], 1 \leq i \leq n\right\rangle \subseteq E_{n}$ by proving that for $k>0,\left[x, y^{k}\right] \in E_{k-1}\left[x,{ }_{k} y\right]$. For $k=1$ it is clear. Assume now that $\left[x, y^{k}\right] \in E_{k-1}\left[x,{ }_{k} y\right]$. If replace $x \rightarrow[x, y]$ then

$$
\left[[x, y], y^{k}\right] \in E_{k}\left[x,{ }_{k+1} y\right] .
$$

By applying the assumption and its consequence to the commutator identity

$$
\left[x, y^{k+1}\right]=\left[x, y^{k}\right][x, y]\left[[x, y], y^{k}\right]
$$

we obtain for $k \geq 0$,

$$
\left[x, y^{k+1}\right] \in E_{k}\left[x,{ }_{k+1} y\right] .
$$

So in view of $(3)$,

$$
w \in\left\langle x,\left[x, y^{i}\right], 1 \leq i \leq n\right\rangle \subseteq E_{n} .
$$

Hence every commutator law is equivalent to a law $w \equiv 1$, where for some $n$, the word $w$ is a product of the Engel commutators $\left[x,{ }_{i} y\right], 1 \leq i \leq n$. By ordering these factors modulo $E_{n}^{\prime}$, we get the law with construction

$$
[x, y]^{k_{1}}\left[x,{ }_{2} y\right]^{k_{2}} \ldots\left[x,{ }_{n} y\right]^{k_{n}} \widetilde{\in} E_{n}^{\prime}, \quad k_{i} \in \mathbb{Z}, n \geq 0 .
$$

\section{The Milnor property and R-laws}

To consider a special kind of laws, we recall the definition of the Milnor property of groups, the name of which was suggested by F. Point in [11].

Definition 2. A group $G$ satisfies the Milnor property if for all elements $g, h \in G$ the subgroup $\left\langle h^{-i} g h^{i}, i \in \mathbb{Z}\right\rangle$ is finitely generated. 
Note that the group $\left\langle h^{-i} g h^{i}, i \in \mathbb{Z}\right\rangle$ is invariant for conjugation by $h$, hence if it is finitely generated then it is equal to $\left\langle h^{-i} g h^{i}, i \in \mathbb{N}\right\rangle$.

The name of the property was motivated by result of Milnor ([8] Lemma 3) who proved that if a finitely generated group $G$ has this property and $A$ is an abelian normal subgroup in $G$ so that $G / A$ is cyclic then $A$ is finitely generated. In 1976 Rosset noticed that the assumption that $A$ is abelian can be dropped and proved the following results which we present in the following Lemma.

Lemma 1. Let $G$ be a finitely generated group satisfying the Milnor property.

(i) Then $G^{\prime}$ is finitely generated.

(ii) If $G / N$ is cyclic then $N$ is finitely generated.

(iii) If $G / N$ is polycyclic then $N$ is finitely generated.

Proof. For (i) and (ii) see ([12] Lemmas 2,3), ([7] Lemma 3, Corollary 4). Note that in [7] the groups satisfying the Milnor property are called restrained. For (iii), if $G / N$ is polycyclic then there is a finite subnormal series with cyclic factors $G=N_{0} \triangleright N_{1} \triangleright \cdots \triangleright N_{m}=N$. Then by means of $m$ successive applications of $(i i)$ we conclude that $N$ is finitely generated.

We introduce a class of laws which we call the $\mathfrak{R}$-laws (restraining laws) because, as we show, every group satisfying the $\mathfrak{R}$-law has the Milnor property (is restrained).

Definition 3. A law is called an $\mathfrak{R}$-law if it implies a law with the following Engel construction where $k_{i} \in \mathbb{Z}, n \geq 1$.

$$
[x, y]^{k_{1}}\left[x,{ }_{2} y\right]^{k_{2}} \ldots\left[x,{ }_{n-1} y\right]^{k_{n-1}}\left[x,{ }_{n} y\right] \widetilde{\in} E_{n-1}^{\prime} .
$$

Example 1. It is clear that an $n$-Engel law $\left[x,{ }_{n} y\right] \equiv 1$ is the $\mathfrak{R}$-law.

Example 2. Each law of the form $x^{k} \equiv 1$ is the $\mathfrak{R}$-law because it implies the law $\left[x, y^{k}\right] \equiv 1$ which in view of (6) implies a law of the form (7).

Theorem 2. A law is an $\mathfrak{R}$-law if and only if every group satisfying this law has the Milnor property.

Proof. We denote $P_{k}=\left\langle x, x^{y^{i}}, 1 \leq i \leq k\right\rangle$ and show that $\left[x,{ }_{k} y\right] \in P_{k-1} x^{y^{k}}$. For $k=1$ it is clear. Assume that $\left[x,{ }_{k} y\right] \in P_{k-1} x^{y^{k}}$, then

$$
\left[x,{ }_{k+1} y\right] \in\left(P_{k-1} x^{y^{k}}\right)^{-1}\left(P_{k-1} x^{y^{k}}\right)^{y} \subseteq P_{k} x^{y^{k+1}} .
$$


It follows for $k \geq 0$ that $E_{k} \subseteq P_{k}$ which implies the equality $E_{k}=P_{k}$, because

$$
E_{k} \subseteq P_{k}=\left\langle x, x^{y^{i}}, 1 \leq i \leq k\right\rangle \stackrel{(3)}{=}\left\langle x,\left[x, y^{i}\right], 1 \leq i \leq k\right\rangle \stackrel{(6)}{\subseteq} E_{k} .
$$

Hence the construction $\left[x,{ }_{n} y\right] \widetilde{\in} E_{n-1}$ is equivalent to $x^{y^{n}} \widetilde{\in} P_{n-1}$, that is

$$
x^{y^{n}} \widetilde{\in}\left\langle x, x^{y}, x^{y^{2}}, \ldots, x^{y^{n-1}}\right\rangle .
$$

We use conjugation by $y^{-n}$, so each $\mathfrak{R}$-law has also construction

$$
x \widetilde{\in}\left\langle x^{y^{-n}}, x^{y^{-(n-1)}}, \ldots, x^{y^{-2}}, x^{y^{-1}}\right\rangle,
$$

and if change $y \rightarrow y^{-1}$ we have

$$
x \widetilde{\in}\left\langle x^{y}, x^{y^{2}}, \ldots, x^{y^{(n-1)}}, x^{y^{n}}\right\rangle .
$$

Let $G$ be a relatively free group, freely generated by $a, b, \ldots$, satisfying an $\Re$-law. Then (10) implies

$$
a \in\left\langle a^{b}, a^{b^{2}}, \ldots, a^{b^{(n-1)}}, a^{b^{n}}\right\rangle,
$$

We conjugate (11) by $b^{-1}$, then

$$
a^{b^{-1}} \in\left\langle a, a^{b}, \ldots, a^{b^{(n-2)}}, a^{b^{(n-1)}}\right\rangle \stackrel{(11)}{\subseteq}\left\langle a^{b}, a^{b^{2}}, \ldots, a^{b^{(n-1)}}, a^{b^{n}}\right\rangle .
$$

By repeating the conjugation by $b^{-1}$ we obtain for all $i>0$,

$$
a^{b^{-i}} \in\left\langle a^{b}, a^{b^{2}}, \ldots, a^{b^{(n-1)}}, a^{b^{n}}\right\rangle .
$$

Similarly, by (9),

$$
a \in\left\langle a^{b^{-n}}, a^{b^{-(n-1)}}, \ldots, a^{b^{-2}}, a^{b^{-1}}\right\rangle .
$$

Conjugation by $b$ gives $a^{b} \in\left\langle a^{b^{-n+1}}, a^{b^{-n}}, \ldots, a^{b^{-1}}, a\right\rangle \stackrel{(13)}{\subseteq}\left\langle a^{b^{-n}}, a^{b^{-n+1}}, \ldots, a^{b^{-1}}\right\rangle$. By repeating conjugation we obtain for all $i>0, a^{b^{i}} \in\left\langle a^{b^{-n}}, a^{b^{(-n+1)}}, \ldots, a^{b^{-1}}\right\rangle$, which, together with (12) finally gives that the subgroup

$$
\left\langle b^{-i} a b^{i}, \quad i \in \mathbb{Z}\right\rangle=\left\langle a^{b^{-n}}, a^{b^{-(n-1)}}, \ldots, a^{b^{-1}}, a, a^{b}, \ldots, a^{b^{n-1}}, a^{b^{n}}\right\rangle
$$

is finitely generated. Since for all elements $g, h$ in any group satisfying the $\Re$-law, the subgroup $\left\langle h^{-i} g h^{i}, i \in \mathbb{Z}\right\rangle$ is an image of $\left\langle b^{-i} a b^{i}, i \in \mathbb{Z}\right\rangle$, we conclude that the $\mathfrak{R}$-law implies the Milnor property. 
Conversely, let $G$ be a relatively free group with free generators $a, b$. If the subgroup $\left\langle b^{-i} a b^{i}, \quad i \in \mathbb{Z}\right\rangle$ is finitely generated then there exists $n$ such that the condition (14) holds. Conjugation by $b^{n}$ implies that

$$
\left\langle b^{-i} a b^{i}, \quad i \in \mathbb{Z}\right\rangle=\left\langle a, a^{b}, a^{b^{2}}, \ldots, a^{b^{2 n}}\right\rangle=\left\langle b^{-i} a b^{i}, \quad i \in \mathbb{N}\right\rangle .
$$

So we have

$$
a^{b^{2 n+1}} \in\left\langle a, a^{b}, a^{b^{2}}, \ldots, a^{b^{2 n}}\right\rangle .
$$

Since each relator on free generators is a law (see [9] 13.21), $G$ satisfies a law with construction of the form (8) which defines the $\Re$-laws.

Theorem 3. A law is an $\mathfrak{R}$-law if and only if every finitely generated group satisfying this law has a finitely generated commutator subgroup.

Proof. If $G$ satisfies an $\mathfrak{R}$-law then by Theorem 2, $G$ has the Milnor property an hence by Lemma $1(i), G^{\prime}$ is finitely generated.

Conversely, let $G$ be a relatively free group defined by a law $w \equiv 1$, with free generators $a, b$ and let $G^{\prime}$ be finitely generated. Then the normal closure of $a$ is equal to $\left\langle b^{-i} a b^{i}, \quad i \in \mathbb{Z}\right\rangle=\langle a\rangle[\langle a\rangle,\langle b\rangle]=\langle a\rangle G^{\prime}$, hence is finitely generated. Then for some $n$ the condition (14) holds. It follows as above, that $G$ satisfies the Milnor property and then by Theorem 2, it satisfies an R-law.

Positive laws are the laws of the form $u\left(x_{l}, x_{2}, \ldots x_{n}\right)=v\left(x_{l}, x_{2}, \ldots x_{n}\right)$, where $u, v$ are distinct words in the free group $\left\langle x_{l}, x_{2} \ldots\right\rangle$, written without negative powers of $x_{l}, x_{2}, \ldots x_{n}$.

Example 3. Each positive law is an $\mathfrak{R}$-law.

Proof. Each positive law implies a binary positive law if substitute $x_{i} \rightarrow x y^{i}$. It was shown by many authors (e.g. [6], [7], [11], [2] p.520) that if a group $G$ satisfies a binary positive law then $G$ has the Milnor property. Thus by Theorem 2, positive laws are the $\mathfrak{R}$-laws.

Example 4. For all prime $p$, the variety $\mathfrak{A}_{p} \mathfrak{A}$, where $\mathfrak{A}$ is the variety of all abelian groups, and $\mathfrak{A}_{p}$ - of all abelian groups of exponent $p$, does not satisfy an $\mathfrak{R}$-law.

Proof. The variety $\mathfrak{A}_{p} \mathfrak{A}$ contains a 2-generator group $W:=C_{p} w r C$, the wreath product of a cyclic of order $p$ group $C_{p}=\langle a\rangle$ and an infinite cyclic group $C=\langle b\rangle$. The commutator subgroup $W^{\prime}$ of this group contains elements $a^{-1} a^{b^{i}}$ for all $i \in \mathbb{Z}$, hence $W^{\prime}$ has an infinite support and cannot be finitely generated. So by Theorem $2, \mathfrak{A}_{p} \mathfrak{A}$ does not satisfy an $\mathfrak{R}$-law. 
A finitely generated residually finite group satisfying either an Engel law or a positive law is nilpotent-by-finite. It was proved for the Engel laws in [14] and for positive laws in [13]. By Examples 2 and 3, the Engel laws and positive laws are the $\mathfrak{R}$-laws. The following lemma extends the statement to the class of $\mathfrak{R}$-laws.

Lemma 2. Every finitely generated residually finite group satisfying an $\mathfrak{R}$ law is nilpotent-by-finite.

Proof. It follows from ([3] Theorem A) that if a law $w \equiv 1$ forces every finitely generated metabelian group satisfying this law to have a nilpotent (of class $c$, say) subgroup of finite index ( $e$, say), then the same holds for every group in the class containing in particular all residually finite groups. Moreover, the parameters $c, e$ depend on the law only.

So it suffices to show that every finitely generated metabelian group satisfying an $\mathfrak{R}$-law is nilpotent-by-finite. Let $G$ be a finitely generated soluble (in particular metabelian) group satisfying an $\mathfrak{R}$-law. By Groves ([5] Theorem C), $G$ is either nilpotent-by-finite or $\operatorname{var} G$ contains a subvariety $\mathfrak{A}_{p} \mathfrak{A}$. Since the latter is not possible in view of Example 4, the proof is complete.

The next property of $\mathfrak{R}$-laws concerns a finite residual $R$ in a group $G$, that is the intersection of all subgroups of finite index in $G$.

Theorem 4. Every finitely generated group $G$ satisfying an $\mathfrak{R}$-law has its finite residual $R$ finitely generated.

Proof. By assumption the group $G / R$ satisfies an $\mathfrak{R}$-law, hence by Theorem 2 it has the Milnor property. Then by Lemma $2, G / R$ is nilpotent-by-finite.

So $G / R$ contains a nilpotent subgroup $H / R$ of finite index. Now, since $|G: H|=|(G / R):(H / R)|<\infty$ and $G$ is finitely generated, both $H$ and $H / R$ are finitely generated. Being a finitely generated nilpotent group, $H / R$ is polycyclic (see [9] 31.12). Since $H / R$ also has the Milnor property, we conclude by Lemma 1 (iii) that $R$ is finitely generated.

\section{$\Re$-laws and locally graded groups}

The common property of the Engel laws and positive laws of being the $\mathfrak{R}$-laws is necessary and sufficient to answer why they force finitely generated locally graded groups satisfying them to be nilpotent-by-finite. 
We recall that a group $G$ is called locally graded if every nontrivial, finitely generated subgroup of $G$ has a proper normal subgroup of finite index. The class of locally graded groups is closed under taking subgroups, extensions and groups which are locally-or-residually 'locally graded'. The class of locally graded groups was introduced in 1970 by S.N.Černikov [4] to avoid groups such as infinite Burnside groups or Ol'shanskii-Tarski monsters.

We can prove now the following

Theorem 5. Every finitely generated locally graded group satisfying an $\mathfrak{R}$ law is nilpotent-by-finite.

Proof. Let $G$ be a finitely generated locally graded group. By Theorem 4, its finite residual $R$ is finitely generated. Then, since $G$ is locally graded, if $R \neq 1$, it must contain a proper subgroup (hence a proper normal subgroup) of finite index $T \subsetneq R$, say. Then by ([9], 41.43), $T$ contains a subgroup $K$ of finite index in $R$ and fully invariant in $R, K \subseteq T \subsetneq R$. Thus $K$ is normal in $G$. Now, since $R / K$ is finite and $G / R$ is nilpotent-by-finite, the isomorphism $(G / K) /(R / K) \cong G / R$ implies that $G / K$ is finite-by-(nilpotentby-finite). Since finite-by-nilpotent group is nilpotent-by-finite, whence $G / K$ is nilpotent-by-finite and then residually finite. It implies $R \subseteq K$, which contradicts to $K \subseteq T \subsetneq R$. Hence $R=1$.

So $G$ is residually finite and by Lemma 2 is nilpotent-by-finite as required.

Moreover, let $\mathfrak{N}_{c}$ denote the variety of all nilpotent groups of class $\leq c$ and $\mathfrak{B}_{e}$ - the variety consisting of all locally finite groups of exponent dividing $e$. (Note that the fact that the class $\mathfrak{B}_{e}$ is actually a variety, is a consequence of Zelmanov's solution of the restricted Burnside problem.) Then by result in [3] (see the proof of Lemma 2) we obtain

Corollary 1. For every $\Re$-law there exist positive integers $c$ and e depending only on the law, such that every locally graded group satisfying this law lies in the product variety $\mathfrak{N}_{c} \mathfrak{B}_{e}$

Note Outside of the class of locally graded groups there are finitely generated groups satisfying $\mathfrak{R}$-laws (in particular, positive laws), which are not nilpotent-by-finite. For example a free Burnside group $B(r, n), r>1$ satisfies the $\mathfrak{R}$-law $x^{n} \equiv 1$. If $n$ is sufficiently large the group is infinite by results of Novikov and Adian (see [1]), hence it is not nilpotent-by-finite. Note also 
that a free finitely generated group satisfying the $\mathfrak{R}$-law $x y^{n}=y^{n} x$ is not nilpotent-by-finite for $n$ sufficiently large.

Another example was given by Ol'shanskii and Storozhev in [10].

Problem Is there an $\mathfrak{R}$-law that implies neither positive nor Engel law?

\section{Acknowledgement}

The author is very grateful to Referee for the valuable remarks.

\section{References}

[1] S. I. Adian, The Problem of Burnside And identities in Groups, Nauka, Moscow, 1975 (Russian), (see also, trans. J. Lennox and J.Wiegold, Ergebnisse der Mathematik und ihrer Grenzgebiete 92, Springer-Verlag, Berlin, 1979).

[2] Robert G.Burns, Olga Macedońska, Yuri Medvedev, Groups Satisfying Semigroup Laws, and Nilpotent-by-Burnside Varieties, J. of Algebra 195 (1997), 510-525.

[3] Burns, R. G., Medvedev, Yu., Group laws implying virtual nilpotence, J. Austral. Math. Soc. 74 (2003), 295-312.

[4] Černikov, S. N., Infinite nonabelian groups with an invariance condition for infinite nonabelian subgroups (in Russian) Dokl. Akad. Nauk SSSR 194, 1970 1280-1283.

[5] Groves, J.R.J., Varieties of soluble groups and a dichotomy of P.Hall, Bull. Austral. Math. Soc. 5 (1971), 391-410.

[6] K.W. Gruenberg, Two theorems on Engel groups, Math. Proc. Cambridge Philos. Soc. 49 (1953), 377-380.

[7] Y. K. Kim and A. H. Rhemtulla, Weak maximality conditions and polycyclic groups, Proc. Amer. Math. Soc. 123 (1995), 711-714.

[8] J. Milnor, Growth of finitely generated solvable groups, J. Diff. Geom. 2 (1968), 447-449. 
[9] H. Neumann, VARIEties of Groups, Springer-Verlag, BerlinHeidelberg-New York 1967.

[10] A.Yu.Ol'shanskii and A.Storozhev, A group variety defined by a semigroup law, J. Austral. Math. Soc. (Series A), 60, (1996), 255-259.

[11] F. Point, Milnor identities, Comm. Algebra 24 (12) (1996) 3725-3744.

[12] S. Rosset, A property of groups of non-exponential growth, Proc. Amer. Math. Soc. 54 (1976), 24-26.

[13] J. F. Semple and A.Shalev, Combinatorial conditions in residually finite groups, I, J.Algebra 157 (1993), 43-50.

[14] J. S. Wilson, Two-generator conditions for residually finite groups, Bull. London Math. Soc 23 (1991), 239-248. 